\title{
LETTER
}

\section{Sex-dependent selection on an autosomal melanic female ornament promotes the evolution of sex ratio bias}

Alexandre Roulin, ${ }^{1 *}$ Res Altwegg, ${ }^{2,3}$ Henrik Jensen, ${ }^{4}$ Ingelin Steinsland ${ }^{5}$ and Michael Schaub ${ }^{6,7}$

\begin{abstract}
Sex-dependent selection often leads to spectacularly different phenotypes in males and females. In species in which sexual dimorphism is not complete, it is unclear which benefits females and males derive from displaying a trait that is typical of the other sex. In barn owls (Tyto alba), females exhibit on average larger black eumelanic spots than males but members of the two sexes display this trait in the same range of possible values. In a 12 -year study, we show that selection exerted on spot size directly or on genetically correlated traits strongly favoured females with large spots and weakly favoured males with small spots. Intense directional selection on females caused an increase in spot diameter in the population over the study period. This increase is due to a change in the autosomal genes underlying the expression of eumelanic spots but not of sex-linked genes. Female-like males produced more daughters than sons, while male-like females produced more sons than daughters when mated to a small-spotted male. These sex ratio biases appear adaptive because sons of male-like females and daughters of female-like males had above-average survival. This demonstrates that selection exerted against individuals displaying a trait that is typical of the other sex promoted the evolution of specific life history strategies that enhance their fitness. This may explain why in many organisms sexual dimorphism is often not complete.
\end{abstract}

\section{Keywords}

Intralocus genetic conflict, melanin-based coloration, sex ratio, sexual selection, sexually antagonistic selection

Ecology Letters (2010) 13: 616-626

\section{INTRODUCTION}

The evolutionary stability of sexual dimorphism and maintenance of genetic variation are issues central to sexual selection theory. Males and females are caught in an evolutionary conflict which stems from their differing reproductive interests which can lead to sexually antagonistic selection. Sex-dependent selection may play an important role in the maintenance of sexually selected ornaments with alleles of the underlying polymorphic genes being beneficial

\footnotetext{
${ }^{1}$ Department of Ecology and Evolution, University of Lausanne, Biophore 1015 Lausanne, Switzerland

${ }^{2}$ South African National Biodiversity Institute, Kirstenbosch Research Centre, P/Bag X7, Claremont 7735, South Africa ${ }^{3}$ Animal Demography Unit, University of Cape Town, Department of Zoology, Rondebosch 7701, South Africa ${ }^{4}$ Department of Biology, Centre for Conservation Biology, Norwegian University of Science and Technology, 7491 Trondheim, Norway
}

\author{
${ }^{5}$ Department of Mathematical Sciences, Centre for Conserva- \\ tion Biology, Norwegian University of Science and Technology, \\ 7491 Trondheim, Norway \\ ${ }^{6}$ Swiss Ornithological Institute, 6204 Sempach, Switzerland \\ ${ }^{7}$ Division of Conservation Biology, Institute of Ecology and \\ Evolution, University of Bern, Baltzerstrasse 6, 3012 Bern, \\ Switzerland \\ *Correspondence: E-mail: Alexandre.Roulin@unil.ch
}


to one sex and detrimental to the other sex leading to intralocus genetic conflict (Chippindale et al. 2001; Bonduriansky \& Chenoweth 2009; Westneat \& Sih 2009). If the two sexes differentially express a sexually selected ornament, individuals displaying a trait that is typical of the other sex will be counter-selected and mechanisms may evolve to enhance their fitness. In the side-blotched lizard (Uta stansburiana) a model that combines game-theoretical methods and population genetic calculations predicts that sexually antagonistic selection will favour females to conditionally bias sex ratio towards sons or daughters with high fitness in relation to their own genotype and the genotype of their mate (Calsbeek \& Sinervo 2002; Alonzo \& Sinervo 2007). Furthermore, natural selection may promote mechanisms to allow male-like females to produce sons with an aboveaverage survival and female-like males to produce daughters with an above-average survival (Calsbeek \& Sinervo 2002, 2003; Day \& Bonduriansky 2004). This would allow these counter-selected phenotypes to reduce the cost of sexually antagonistic selection. The study of sex-dependent selection is currently a popular topic (Bonduriansky \& Chenoweth 2009) and particular attention is devoted to its effect on genome evolution and on the expression of genes located on autosomes vs. sex chromosomes (Mank 2009). What is currently needed is to integrate field studies showing the phenotypic consequences of sex-dependent selection combined with an analysis of the effect on the frequency and expression of the underlying genes. To this end, the study of melanin-based colour traits is promising because interindividual variation in colouration is often heritable and candidate genes identified (e.g. Mundy 2005; Ducrest et al. 2008). Our aim in the present study is to investigate the phenotypic consequences of sex-dependent selection.

The barn owl is appropriate to investigate the evolutionary implications of sex-dependent selection because females display on average larger black eumelanic spots on their plumage than males, a trait that signals female genetic quality (Roulin 2004a) and is a criterion in male mate choice (Roulin 1999; Roulin \& Altwegg 2007). Interestingly, members of the two sexes exhibit eumelanic spots in the same range of possible diameters begging the question of whether typical females and those displaying spots of a size which is typical to males adopt different life history strategies (and similarly for males). To examine this proposition, we need to carry out a thorough study on sex-dependent selection.

Previous studies showed that spot diameter is positively correlated with survival and offspring quality in females rather than in males (Roulin 2004a; Roulin \& Altwegg 2007), and hence we predict that spot size is directionally selected in females rather than in males. If this is the case, two results had to be verified at the phenotypic and genetic level. First, as the expression of black spots is heritable, directional selection had to lead to microevolution with spot diameter increasing in size along the 12-year study period. Second, in birds females are heterogametic $(\mathrm{ZW})$ and males homogametic (ZZ), and thus selection would have a stronger effect on dominant autosomal genes than genes located on sex chromosomes (Charlesworth et al. 1987). Because recent studies suggest that in birds sexually antagonistic genes are dominant and over-represented on autosomes (Ellegren \& Parsch 2007; Mank \& Ellegren 2009), we predict a change in the mean breeding value only of the underlying autosomal genes over the study period. If these two predictions are supported, we will investigate how counter-selected malelike females (and potentially counter-selected female-like males) can enhance their fitness. Male-like females may bias sex ratio towards high-quality sons and female-like males bias sex ratio towards high-quality daughters.

\section{MATERIALS AND METHODS}

The study was carried out between 1996 and 2007 in western Switzerland where 20-80 pairs breed each year in 110 nest-boxes put up in barns over an area covering $30 \times 7 \mathrm{~km}$. In this study, we report a brief summary of the methods. More details can be found in Appendix S1.

\section{Survival and age-specific probability of first reproduction}

We analysed survival using combined capture-recapture and ring-recovery models (Lebreton et al. 1992; Burnham 1993). Our analysis builds on earlier work (Altwegg et al. 2007), and the global model included factors that we have found earlier to be important, in particular sex and age. The modelling approach used in this study assumes that all individuals within a group (sex and age class) have similar survival, recapture, recovery and fidelity rates (homogeneity assumption). A goodness-of-fit test showed that our data met the homogeneity assumption $(\hat{c}=1.076, \mathrm{SE}=0.013)$. We fitted all models using program MARK 5.1 (White \& Burnham 1999) and used the sample-size adjusted Akaike's Information Criterion (AICc) for model selection. After determining the best model structure excluding spot diameter, we examined the effects of spot diameter on survival by adding this trait as an individual covariate. We used a two-state extension of the same capture-markrecapture methods to estimate the age-specific probability of first reproduction, and the relationship between spot diameter and this fitness component. The 'local juvenile survival rate' is 0.05 for females and 0.08 for males (Altwegg et al. 2007).

\section{Estimating selection gradients}

Selection gradients measure the change in fitness as a result of a change in a particular trait. In this article, we 
use the logarithm of the dominant eigenvalue of a population projection matrix $[\log (\lambda)]$ as a measure of fitness. It is the long-run population growth rate that (a group of) individuals with a particular trait value would have. The sensitivity of $\log (\lambda)$, i.e. the partial derivative of $\log (\lambda)$ (see Caswell 2001) to change in standardized (to mean zero and unit standard deviation) spot diameter is then equivalent to the selection gradient on spot diameter (or on genetically correlated traits; Van Tienderen 2000; Coulson et al. 2003). For the matrix projection model, we assume a pre-breeding census and a 1-year projection interval. We explicitly incorporate both males and females because we were interested in selection acting on each sex. The model is:

$n_{t+1}=M n_{t}$

where $n_{t}$ is a vector specifying the number of individuals in each stage (age class, sex) at time $t$, and $M$ is the population projection matrix:

$M=\left(\begin{array}{cccc}\alpha_{\mathrm{f}} F_{\mathrm{df}} & F_{\mathrm{df}} & \alpha_{\mathrm{m}} F_{\mathrm{dm}} & F_{\mathrm{dm}} \\ s_{\mathrm{yf}} & s_{\mathrm{f}} & 0 & 0 \\ \alpha_{\mathrm{f}} F_{\mathrm{sf}} & F_{\mathrm{sf}} & \alpha_{\mathrm{m}} F_{\mathrm{sm}} & F_{\mathrm{sm}} \\ 0 & 0 & s_{\mathrm{ym}} & s_{\mathrm{m}}\end{array}\right)$

This matrix consists of four blocks of dimension $2 \times 2$. The upper left block describes the dynamics of the female segment of the population, the lower right block describes the male segment, the lower left is the females' contribution to sons, and the upper right is the males' contribution to daughters. In this study, $\alpha_{\mathrm{f}}$ and $\boldsymbol{\alpha}_{\mathrm{m}}$ are the probabilities of starting to breed at age 1 for females and males, respectively. Adult females and males survive at rates $s_{\mathrm{f}}$ and $s_{\mathrm{m}}$, and survival of 1-year-old females and males are $s_{\mathrm{yf}}$ and $s_{\mathrm{ym}}$. The terms denoted by $F$ describe production of daughters (subscript d) and sons (subscript s) by females (subscript $\mathrm{f}$ ) and males (subscript $\mathrm{m}$ ). To give an example of how these terms are calculated:

$F_{\mathrm{df}}=s_{\mathrm{jf}}\left(k_{\mathrm{df}}+p l_{\mathrm{df}}\right) \times \min \left[\frac{n_{\mathrm{f}}}{n_{\mathrm{f}}+n_{\mathrm{m}}}, \frac{n_{\mathrm{m}}}{n_{\mathrm{f}}+n_{\mathrm{m}}}\right]$

where $s_{\mathrm{jf}}$ is survival of juvenile females, $k_{\mathrm{df}}$ is the number of daughters produced by females during the first brood, $p$ is the probability of producing a second brood, and $l_{\mathrm{df}}$ is the number of daughters produced by females in a second brood. The last term is the birth function, where $n_{\mathrm{f}}$ is the number of females and $n_{\mathrm{m}}$ the number of males. We chose this particular birth function because a successful brood in barn owls needs the full commitment of both parents. The rarer sex limits reproduction in our model. The other fertility functions $\left(F_{\mathrm{dm}}, F_{\mathrm{sf}}\right.$ and $\left.F_{\mathrm{sm}}\right)$ were identical to eqn (3) with $s_{j}$, $k$ and $l$ replaced by the appropriate terms. Our model does not address the possibility of assortative mating with respect to plumage. This is justified by the weak correlation between female and male spot diameter among pairs in our data set $(n=468, r=0.065)$.

This matrix model is non-linear. $F$ depends on the population vector $n$, making it frequency-dependent but not density-dependent. Its dynamics thus depend on the sex ratio, but are independent of the absolute values of $n$. We used simulations performed in the program $\mathrm{R}$ 2.9.0 (R Development Core Team 2009) to estimate the population growth rate $\lambda$ and the stable sex ratio (Morris $\&$ Doak 2002). We started the simulations with a total population size of 1 , and recorded the population growth rate and sex ratio after iteration 100 , at which point a stable age and sex distribution had always been reached. We then estimated the relationship between male and female spot diameter on each of the parameters of this model. In these analyses, we always included spot diameter for both sexes simultaneously to obtain partial regression coefficients while correcting for the other effects (Lande \& Arnold 1983).

Linear standardized selection gradients are calculated as the partial regression coefficient of a trait, standardized to unit variance, on a fitness component while holding other effects constant (Lande \& Arnold 1983). The population growth rate $\log (\lambda)$ computed from eqn (2) when all fitness components are set to the population mean gives a measure of mean fitness (Metz et al. 1992). Let $\log \left(\lambda_{\mathrm{D}}\right)$ be the predicted fitness of an individual that differs in plumage from the population mean by one standard deviation. $\log (\lambda)-\log \left(\lambda_{\mathrm{D}}\right)$ is then a measure of selection equivalent to a selection gradient, but using a fitness measure that is based on the whole life cycle. We calculated the confidence interval for this measure of selection using parametric bootstrap methods. For each of the partial regression coefficients, we drew 10000 random values from a normal distribution (on the logit scale for survival, probability of first reproducing at age 1 and probability of producing a second clutch) with mean and variance equal to the observed values. With these values, we reconstructed 10000 matrices and calculated $\log \left(\lambda_{\mathrm{D}}\right)$ for each of them. The limits of the confidence interval are the 2.5th and 97.5th percentiles of $\log (\lambda)-\log \left(\lambda_{\mathrm{D}}\right)$.

\section{Quantitative genetics}

We used an 'animal model' to estimate heritabilities of spot diameter. The animal model is a mixed-model approach that uses information on the relatedness between individuals in a pedigree, combined with phenotypic data on these individuals to estimate quantitative genetic parameters (Kruuk 2004). Here, we used a Bayesian approach (O'Hara et al. 2008) to estimate the parameters of interest. We extended the Bayesian animal model framework developed by Steinsland 
\& Jensen (in press) to estimate simultaneously the autosomal and Z-linked heritability of spot diameter. We did not consider a model of inheritance on the much smaller and less genetically variable W-chromosome (Ellegren 2007) which is transmitted only between mother and their daughters because with respect to spot diameter sons resemble their father more than daughters resemble their mother (Roulin \& Dijkstra 2003). Furthermore, the animal model accounted for any environmental effects due to year of hatching and rearing environment (i.e. cross-fostering) in our analyses. The deviance information criterium (DIC) was used to compare different models (Spiegelhalter et al. 2002). In this model selection framework, the model with lowest DIC is regarded as the 'best', and models receiving a DIC 3-7 higher than the 'best' have considerably less support. Finally, the Bayesian animal model was used to estimate the mean and uncertainty of each cohort's breeding values (autosomal and Z-linked). We included year as random factor in the model to account for any systematic environmental variation across years (Postma 2006). Using a Bayesian animal model framework we accounted for the uncertainty of estimated variance components as well as the uncertainty and non-independence of individual breeding values of birds within a cohort when calculating the mean cohort breeding value and its uncertainty. Furthermore, because we estimated the mean breeding value within each cohort directly instead of taking the mean of estimated individual breeding values within the cohort we avoided problems related to the use of individual breeding values (see e.g. Hadfield et al. 2010). These estimates were then used to test whether any phenotypic changes across the 12-year study period were reflected in genetic changes on autosomes or on $\mathrm{Z}$ chromosomes. When Bayesian procedures are used to estimate any temporal changes the results are more robust than if estimated by (restricted) maximum likelihood methods (Sorensen et al. 1994; Hadfield 2008; Hadfield et al. 2010).

As extra-pair paternity is rare in the barn owl (one out of 211 offspring was not sired by the male that was feeding it, Roulin et al. 2004) the pedigree was constructed by assuming that the social parents were the biological parents. The pedigree used in the analyses consisted of a total of 3264 individuals that hatched between 1987 and 2007, spanning up to eight generations. Sex was known for all individuals in the pedigree. To enable estimation of the Z-specific heritability, birds that had only one known parent (167 with known mother only, 12 with known father only) were assumed to have no known parents. Hence, both parents were known for $76 \%$ of the individuals in the pedigree; the rest had no known parents and comprised the founder population in the pedigree. The pedigree included 275 different fathers and 317 different mothers, who produced on average 9.0 (median 6; range 1-44) and 7.8 (median 6; range 1-51) offspring each, respectively. Twenty percent of the mothers and $30 \%$ of the fathers in the pedigree produced offspring for at least three seasons. Furthermore, average lineage depth was 1.8 generations for birds with both parents known (range 1-8), and generation time $T$ was 3.6 years (Lande et al. 2003). Consequently, the connectedness of the pedigree was fairly high, so that estimates of breeding values should be relatively accurate, and separation of year effects into environmental and genetic parts may be possible (Postma 2006). In the quantitative genetic analyses, we used individual spot diameters that were adjusted to the diameter in the first year of life (see below). The eumelanic spots are expressed already at the nestling stages explaining why we could measure spot diameter in a large number of individuals (2 711 in the pedigree); 1297 of these were males and 1414 were females. In order to avoid any problems with unequal phenotypic variances of spot diameter between sexes, the spot diameters were standardized within sex to have mean 0 and variance 1 . To examine whether the heritability of spot diameter differed between the sexes, we assumed that spot diameter consists of two separate traits, one expressed only in males and one expressed only in females (Jensen et al. 2003).

\section{RESULTS AND DISCUSSION}

\section{Sex-dependent selection}

We examined in the two sexes the association between spot diameter and the most important fitness component, juvenile survival from fledging to the first year of age (Altwegg et al. 2007). We used combined capture-mark-recapture and dead recovery methods (Lebreton et al. 1992; Burnham 1993; Altwegg et al. 2007) with a dataset including 2675 individuals captured and recaptured alive between 1996 and 2007. Of these, 1047 females and 991 males were ringed as nestlings, 149 females and 211 males as yearlings, and 102 females and 75 males as older birds. We also included dead recoveries to estimate survival independently of emigration (Burnham 1993; Altwegg et al. 2003) which is sex-specific with females and males moving on average 23 and $12 \mathrm{~km}$ between the natal nest and the site where they bred in their first year of life, respectively (Roulin \& Altwegg 2007); 126 ringed owls were found dead within our study area and 55 outside. The diameter of eumelanic spots was significantly positively associated with survival of juvenile females but not significantly negatively associated with survival of juvenile males (Tables 1 and 2; Fig. 1a). Model selection based on $\mathrm{AIC}_{\mathrm{c}}$ showed that the model with sex-specific effects of spot diameter on survival was 29 times better supported by the data than the model assuming equal effects of spot size in both sexes (comparing models 9 and 10 of Table S1).

To examine whether the observed selection acting at the juvenile stage had to result in a microevolutionary change in 
Table 1 Model selection for barn owl survival probability (S) in relation to mean spot diameter

\begin{tabular}{|c|c|c|c|c|c|c|}
\hline & Model & $\mathrm{AICc}$ & Delta AICc & AICc weights & $K$ & Deviance \\
\hline 1 & $S_{\text {Juvenile }} \sim$ spot diameter $\times$ sex & 3650.039 & 0.000 & 0.832 & 46 & 3558.039 \\
\hline 2 & No individual covariates & 3654.750 & 4.712 & 0.079 & 44 & 3566.750 \\
\hline 3 & $S_{\text {Adult }} \sim$ spot diameter $\times$ sex & 3654.837 & 4.798 & 0.076 & 46 & 3562.837 \\
\hline 4 & $S_{\text {Yearling }} \sim$ spot diameter $\times$ sex & 3658.296 & 8.258 & 0.013 & 46 & 3566.296 \\
\hline
\end{tabular}

Our base model was the most parsimonious model excluding spot diameter and allowed for various effects on survival, recapture, recovery and fidelity probabilities (see Appendix S1 for details). We based model selection on the sample-size adjusted Akaike's Information Criterion (delta AICc), where a smaller value indicates a better model. The AICc weights give the relative support for a model compared to the other models in the set, and $\mathrm{K}$ is the number of parameters estimated. The effect of spot diameter was allowed to vary between the sexes (interaction between spot diameter and sex), and we examined effects on one age class at the time.

mean spot diameter in our population, we estimated the strength and direction of selection calculated over the whole life cycle in the two sexes (Table 2). The sensitivity of the population growth rate $\lambda$, obtained from a population projection matrix (Metz et al. 1992), to changes in standardized spot diameter is equivalent to the selection gradient on that trait (Van Tienderen 2000; Coulson et al. 2003; Altwegg et al. 2007). For this analysis, we used data collected between 1996 and 2007 on spot diameter measured in 578 breeding females and 550 breeding males, as well as in 1085 nestling females from 481 broods and 1072 nestling males from 476 broods. The results show that large-spotted females were significantly positively selected, while large-spotted males were weakly but non-significantly counter-selected (Table 2). Our results thus suggest that our population is not at equilibrium, otherwise variation in spot size would be rapidly depleted. Directional selection on female spot size is probably the outcome of a combination of sexual selection exerted by males (Roulin 1999) and of indirect selection because female spot size is positively associated with offspring quality (Roulin 2004a) and female survival. Quantifying the relative importance of these effects is complex and beyond the scope of our paper (e.g. Jensen et al. 2008).

\section{Microevolutionary effects of sex-dependent selection on quantitative genetics}

For selection to result in evolutionary change, at least some variation in spot diameter must have a genetic basis. In birds, females are heterogametic $(\mathrm{ZW})$ and males homogametic (ZZ), and earlier analyses have suggested that some of the genes encoding for spot size are Z-linked (Roulin \& Dijkstra 2003). From theory (Rice 1984), we expect that when selection is particularly intense in females, autosomal genes are exposed to selection half of the time whereas $\mathrm{Z}$ linked dominant genes are exposed to selection only onethird of the time. Selection will thus act more strongly on autosomal genes than on those located on the sex chromosomes (Charlesworth et al. 1987). Accordingly, recent studies suggest that sexually antagonistic genes follow a dominant mode of inheritance in birds and that genes beneficial for females are over-represented on autosomes (Ellegren \& Parsch 2007; Mank \& Ellegren 2009). Consequently, we predict that directional selection on female spot diameter mainly results in a change in the mean breeding value of the underlying autosomal genes. We used an animal model in a Bayesian framework to estimate the heritability of spot diameter (Kruuk 2004; O’Hara et al. 2008; Steinsland \& Jensen in press).

Comparison of DIC for Bayesian animal models where only autosomal inheritance of spot size was assumed strongly suggested that there was no effect of the rearing environment, and that the model including year of hatching and additive genetic variance was the best (see models $1-5$ in Table S2). According to the best model (i.e. model 5 in Table S2) total phenotypic variation in spot diameter was dominated by additive genetic variance, with an overall heritability of 0.607 (95\% CI: 0.560 to 0.655$)$, and a small effect of the year of hatching $\left(V_{\mathrm{Y}} / V_{\mathrm{P}}=0.093,95 \% \mathrm{CI}\right.$ : 0.055 to 0.130 ). The estimates are the posterior means and $95 \%$ credibility intervals (CI) for the variance components. The heritability was higher in males $\left(b^{2}=0.823,95 \% \mathrm{CI}\right.$ : 0.745 to 0.890$)$ than in females $\left(b^{2}=0.528,95 \%\right.$ CI: 0.480 to $0.585)$, and DIC of the two models showed that the male model of inheritance was much better than the female model of inheritance (see models 8-9 in Table S2). Furthermore, the analyses suggested that the year of hatching explained almost twice as much of the phenotypic variance in females $\left(V_{\mathrm{Y}} / V_{\mathrm{P}}=0.103,95 \% \mathrm{CI}: 0.055\right.$ to $0.145)$ than in males $\left(V_{\mathrm{Y}} / V_{\mathrm{P}}=0.059,95 \% \mathrm{CI}: 0.005\right.$ to $0.110)$.

In birds, the $\mathrm{Z}$ chromosome is transferred from fathers to both sons and daughters, whereas mothers transfer their $\mathrm{Z}$ chromosome to sons only. Consequently, the higher heritability of spot diameter in males suggests that some of the genes affecting this trait are located on the $\mathrm{Z}$ chromosome. However, higher heritability in one sex 

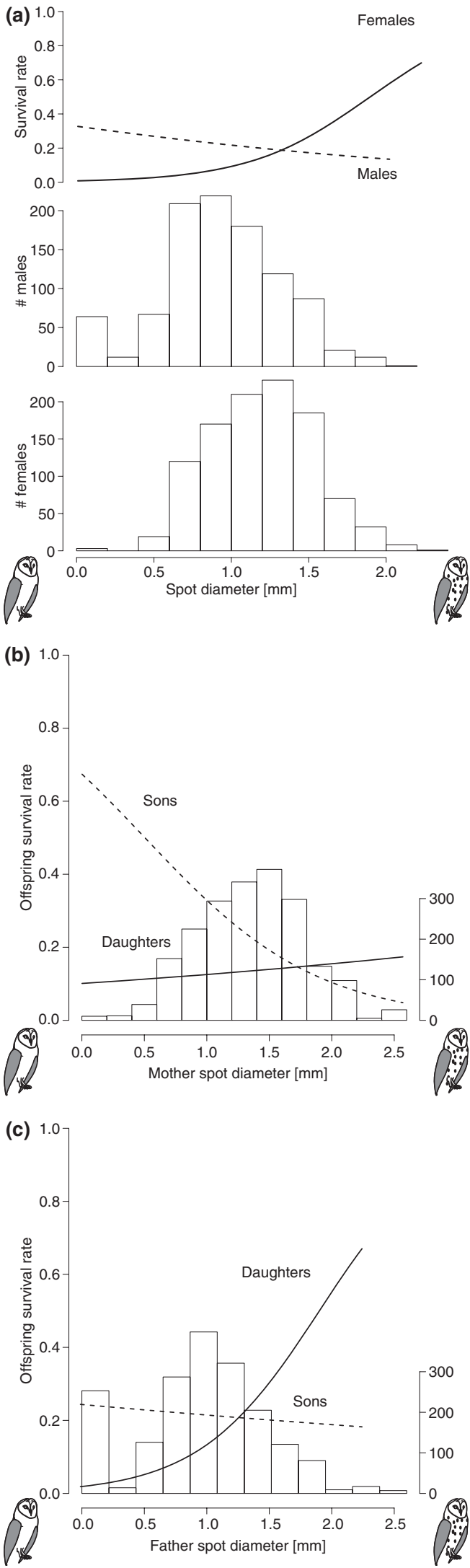

Figure 1 (a) Survival rate of juvenile female (solid line) and male (broken line) barn owls in relation to the diameter of their eumelanic spots. The distribution of observed spot diameters is given as histograms for both sexes below the panel. (b and c) Survival rate of juvenile female (solid line) and male (dashed line) barn owls in relation to their parents' spot diameter (panel b for mother, panel c for father). Survival rate is the probability that individuals survive between fledging and the first year of age. The histograms show the distribution of spot size in our sample.

compared with the other can also be due to sex-specific effects of environmental variation (Jensen et al. 2003), as suggested by differences in the effects of year of hatching. Thus, although our results are suggestive, we cannot conclude on the basis of sex-specific estimates of heritability that any of the genes affecting spot diameter are sex-linked.

We extended the above Bayesian animal model framework to estimate Z-chromosome-specific and autosomal heritabilities and breeding values simultaneously. A comparison of DIC for models with only autosomal inheritance, only Zchromosomal inheritance and both modes of inheritance clearly showed that the model with both autosomal and Zchromosomal inheritance of spot size was best (see models 5 , 6 and 7 in Table S2). The posterior distributions of the heritabilities from the best model (i.e. model 7 in Table S2) are given in Fig. 2. The posterior means and 95\% credibility intervals (CI) were $h_{\mathrm{AZ}}^{2}=0.702(95 \% \mathrm{CI}$ : 0.675 to 0.730$)$, $b_{\mathrm{A}}^{2}=0.570(95 \% \mathrm{CI}: 0.540$ to 0.600$)$ and $b_{\mathrm{Z}}^{2}=0.132$ (95\% CI: 0.120 to 0.145 ). Hence, spot diameter is partly sex-linked as $13 \%$ of its phenotypic variance was caused by variation in genes on the $Z$ chromosome, whereas $57 \%$ of its phenotypic variance was due to variation in genes on autosomes. The remaining variation in spot diameter could be due to non-additive genetic effects, unmeasured environmental effects and measurement errors (Kruuk 2004).

The increase in spot diameter in our population over the study period (Fig. 3a) was reflected in an increase in its estimated mean autosomal breeding values from the 1996 to the 2007 cohort as predicted: the posterior distribution of the mean difference in autosomal breeding values of the two cohorts was 0.140 (95\%CI: 0.009 to 0.270 ; Fig. 3b). In contrast, the mean Z-linked breeding values for spot diameter did not change significantly over the study period: the posterior distribution of the mean difference in Z-linked breeding values of the two cohorts was -0.004 ( $95 \% \mathrm{CI}$ : -0.069 to 0.059 ; Fig. 3b). Although, we cannot exclude the possibility that the increase in autosomal breeding values was a result of genetic drift and not a result of the strong selection acting on females, our results show that the population genetic structure changed substantially over the course of the study according to our above results in the selection analyses. A review of correlative studies performed 
Table 2 Relationship between spot diameter and a suite of fitness components in barn owls, and the selection gradient on overall fitness

\begin{tabular}{|c|c|c|c|c|}
\hline & Trait value & SE & Partial coefficient & SE \\
\hline \multicolumn{5}{|l|}{ Females } \\
\hline Probability of breeding at 1 year of age & 1.000 & $<0.001$ & 1.257 & 2.469 \\
\hline Juvenile survival & 0.265 & 0.055 & 1.048 & 0.387 \\
\hline Yearling survival & 0.591 & 0.048 & 0.128 & 0.190 \\
\hline Adult survival & 0.639 & 0.061 & 0.167 & 0.133 \\
\hline Number of daughters fledged & 2.111 & 0.069 & -0.003 & 0.006 \\
\hline Number of sons fledged & 2.119 & 0.114 & 0.004 & 0.007 \\
\hline logit(probability of producing 2 nd brood) & -2.437 & 0.376 & 0.044 & 0.028 \\
\hline \multicolumn{5}{|l|}{ Males } \\
\hline Probability of breeding at 1 year of age & 0.910 & 0.089 & -1.060 & 0.895 \\
\hline Juvenile survival & 0.397 & 0.054 & -0.232 & 0.186 \\
\hline Yearling survival & 0.725 & 0.038 & -0.009 & 0.208 \\
\hline Adult survival & 0.764 & 0.038 & 0.174 & 0.123 \\
\hline Number of daughters fledged & 2.111 & 0.069 & 0.017 & 0.006 \\
\hline Number of sons fledged & 2.119 & 0.114 & -0.011 & 0.005 \\
\hline logit(probability of producing 2nd brood) & -2.842 & 0.292 & 0.035 & 0.038 \\
\hline Overall selection gradient: change in females & & & 1.695 & $\mathrm{CI}=0.536 / 2.874$ \\
\hline Overall selection gradient: change in males & & & -0.016 & $C I=-0.115 / 0.049$ \\
\hline
\end{tabular}

The table shows mean trait values in the population (trait value) and its standard error (SE). The partial regression coefficients of spot diameter on each fitness component in both sexes are shown with standard errors. Bold type indicates values significantly different from zero. The last two lines of the table show the estimated selection gradients on spot diameter, integrated over the life cycle. The upper values examine the fitness effects of a change in spot diameter in females only, while the lower values refer to males only. We give $95 \%$ confidence intervals (CI) calculated by bootstrap methods.

in other bird species displaying melanin-based colour traits showed that changes in mean population colour values over time are frequent (Roulin 2004b).

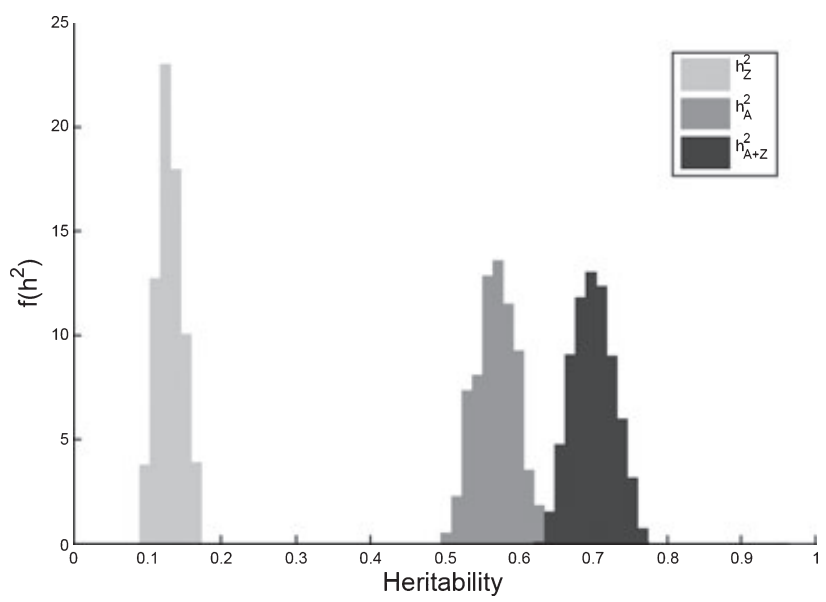

Figure 2 The posterior distribution of the Z-linked heritability (in light grey), autosomal heritability (in dark grey), and sum of Z-linked and autosomal heritability (in black) of spot diameter in the barn owl. The posterior distributions of heritabilities were calculated from a Bayesian animal model (see Steinsland \& Jensen in press) including an autosomal and a Z-linked additive genetic component to spot diameter.

\section{Effect of sex-dependent selection on the evolution of sex ratio}

Selection at the juvenile stage was particularly strong against male-like small-spotted females and to a lesser extent against female-like large-spotted males (Fig. 1a; Table 2). We predicted that counter-selected male-like small-spotted females bias brood sex ratio towards sons, because these may have a higher survival probability than daughters. Similarly, when the father is small-spotted, we expect the brood sex ratio to be biased towards sons, to avoid producing daughters who may have a low survival probability. We do not necessarily predict that female-like males produce more daughters than sons because in the barn owl females are bigger than males and thereby more costly to produce. Using data on offspring sex collected between 1996 and 2007, we estimated the relationship between parental spot diameter and the sex of 3173 nestlings in 640 broods. We used generalized linear models with a logit link function and binomial errors with the response being the sex of the offspring ( 0 for female, 1 for male). We initially included brood identity, female identity, and male identity as random effects to account for possible non-independence among data points. However, these random effects turned out to be very small and the final results are from computationally simpler models with fixed effects only. As 
(a)

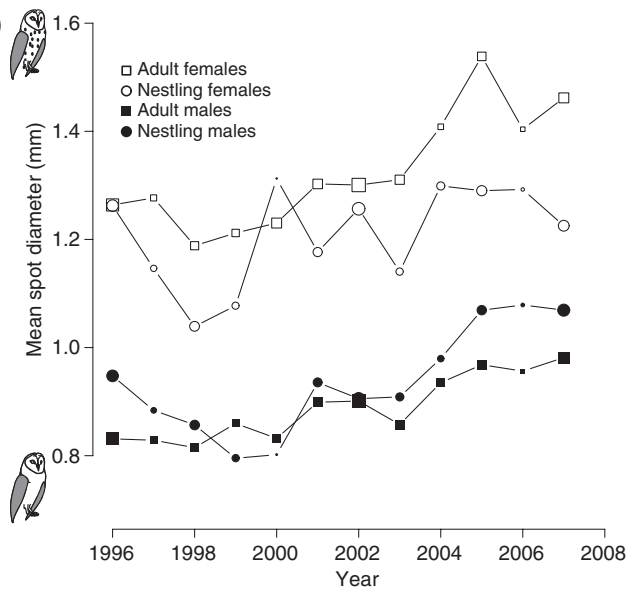

(b)

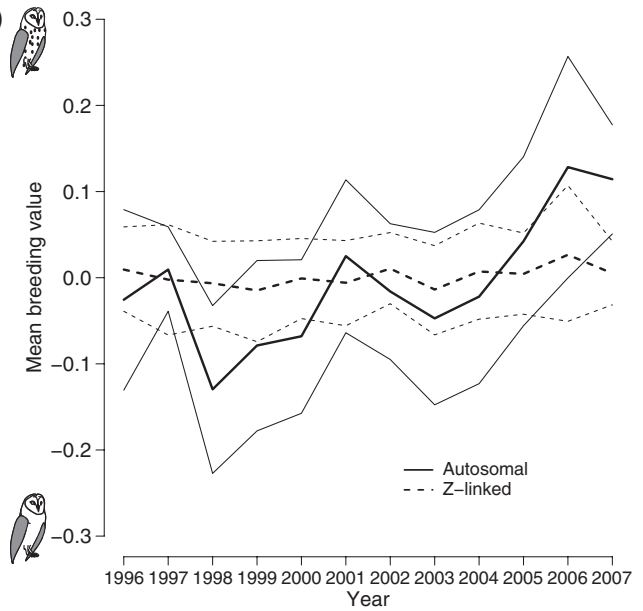

Figure 3 (a) Mean annual spot diameter of female and male breeding barn owls and nestlings between 1996 and 2007. For nestlings, we first calculated mean sibling values and then mean annual values based on all recorded families. The size of the symbols is proportional to sample size, which ranged from 7 to 78 . The Pearson correlation between mean spot diameter and year was significant in adult females $(r=0.83, n=12$ years, $P<0.0001)$, adult males $(r=0.91, P<0.0001)$ and nestling males $(r=0.74$, $P=0.006)$, but not in nestling females $(r=0.46, P=0.14)$. Mean $( \pm \mathrm{SE})$ spot diameter across the whole study period is $1.32 \pm 0.03 \mathrm{~mm}$ for breeding females, $0.89 \pm 0.02 \mathrm{~mm}$ for breeding males, $1.21 \pm 0.03 \mathrm{~mm}$ for nestling females and $0.94 \pm 0.03 \mathrm{~mm}$ for nestling males. (b) Mean (heavy lines) estimated autosomal (solid line) and Z-linked (dashed line) breeding values for spot diameter in nestling barn owls from the cohorts 1996-2007. This figure is constructed from the estimated mean breeding values for cohorts, and thus estimated from the actual data given by the pedigree. The thin lines indicate the $95 \%$ credibility intervals.

sex ratio bias can vary with hatching rank (Blanco et al. 2002), we included this factor and its interaction with parents' spot diameter as explanatory variables. We could investigate relationships between sex ratio and spot diameter of both the father and mother because within pairs spot diameters of the female and male partners were not correlated. The three-way interaction between mother spot diameter, father spot diameter and hatching rank was significant $(z=-2.03, P=0.04)$, as were the two-way interactions between rank and both parents' spot diameter $(P<0.027)$, and all three main effects $(P<0.031)$. The probability of an offspring being male was highest when both parents were small-spotted, and lowest when one of the parents was large-spotted, especially among first-hatched offspring in a brood (Fig. 4a). For subsequent offspring, the pattern tended to vanish (Fig. $4 \mathrm{~b}$ shows the fitted values for an offspring of median rank). As first-hatched chicks rarely die before fledging (Roulin 2002), sex ratio bias probably occurred before hatching.
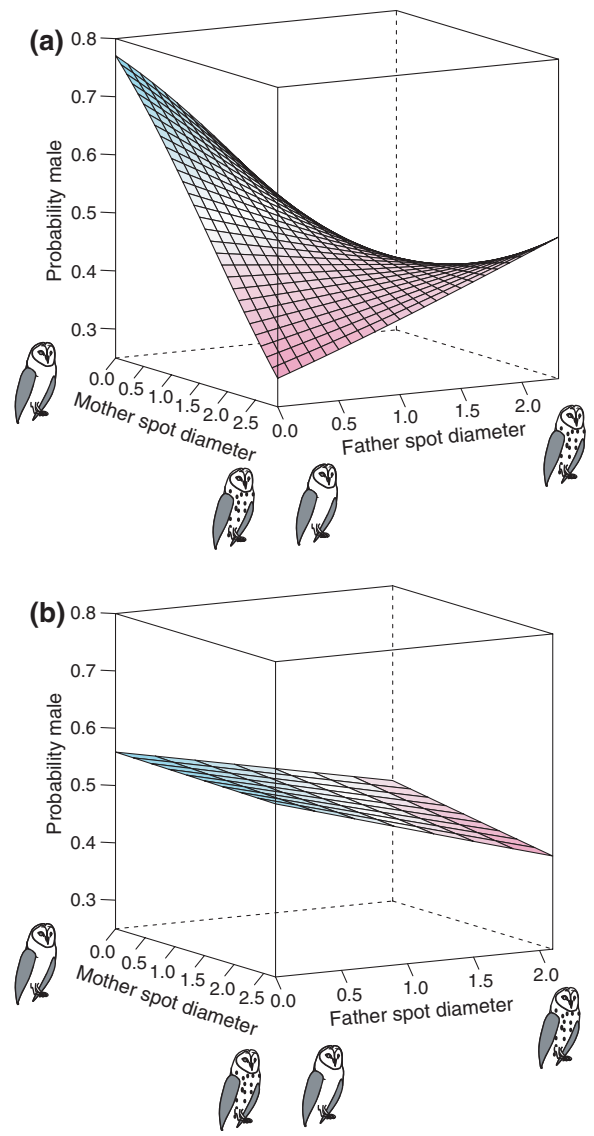

Figure 4 The probability that barn owl offspring were male in relation to spot size of their biological mother and father. The relationship between of parents' spot size and offspring sex dependent on hatching rank, and we therefore show the fitted values for first-born (panel a) and third-born (i.e. median rank; panel b) nestlings. 


\section{Sex ratio bias is adaptive}

Variation in spot diameter is mainly due to autosomal genes, and thus juvenile survival had to be associated with spot diameters measured in both biological parents unless there is a maternal or paternal effect or an effect of Z-linked genes. Our results show that the relationship between parental spot diameter and juvenile survival depends on both the sex of the parents and of the offspring. Spot diameter measured in mothers was significantly negatively associated with the survival of their sons (similar survival analyses as above; logit slope -0.61 , 95\%CI: -1.16 to -0.06 ; Fig. 1 b; Model 17 in Table S1), while spot diameter in fathers was significantly positively associated with the survival of their daughters (logit slope 1.00, 95\%CI: 0.28 to 1.71; Fig. 1c; Model 15 in Table S1). The relationships between maternal spot diameter and their daughters' survival and between paternal spot diameter and their sons' survival were not significant (mother-daughter: $0.102,95 \%$ CI: -0.58 to 0.78 ; fatherson $-0.08,95 \%$ CI: -0.74 to 0.58 ; Fig. $1 \mathrm{~b}, \mathrm{c})$. These sex ratio biases were thus adaptive because male-like females biased sex ratio towards sons who have an above average survival, and because female-like males biased sex ratio towards daughters who have an above average survival.

To demonstrate that these sex-specific survival effects were heritable and thus not inflated by rearing conditions that could be associated with spot diameter, we performed cross-fostering experiments. In 10 of the 12 years, we swapped eggs or hatchlings between randomly chosen pairs of nests (Roulin 1999, 2004a; Roulin \& Altwegg 2007). In this way, we could relate the juvenile survival of 808 nestlings raised in 347 foster broods to the spot diameter of their biological parents. This analysis yielded similar results as those above (mother-son: $-0.72,95 \% \mathrm{CI}:-1.43$ to 0.001 ; father-daughter: $0.88,95 \% \mathrm{CI}: 0.04$ to 1.71$)$. Therefore, the higher survival of small-spotted compared to large-spotted males (Fig. 1a) is a property passed on by their mother (Fig. 1b) either genetically or as a maternal effect via the egg. Similarly, the higher survival of large-spotted compared to small-spotted females (Fig. 1a) is a property transmitted by their father (Fig. 1c).

The selection analysis showed that heavily spotted females are strongly positively selected, while heavily spotted males are only weakly negatively selected (Table 2). This weak negative selective pattern might be explained by the fact that female-like males produced more daughters than typical males (Table 2) and that these daughters have an above-average survival (Fig. 1c). Female spot diameter was less related to the number of male or female offspring (Table 2). Thus, negative selection on large-spotted males might be counter-balanced by the production of daughters showing a large selective advantage.

\section{CONCLUSION}

Sex-dependent selection on fitness has rarely been tested in natural populations (Jensen et al. 2008; review in Bonduriansky \& Chenoweth 2009). We found some evidence for such a selection pattern on a heritable melanin-based ornament in the barn owl. On average, females display larger eumelanic spots than males, and selection at the juvenile stage strongly favours females with large spots and weakly favours males with small spots (Fig. 1; Table 2). Sex-dependent selection on a polymorphic ornament has promoted the evolution of sex ratio biasing mechanisms (Fig. 4) and sex-specific survival effects (Fig. 1b, c) that allow female-like males and male-like females to improve their fitness. As female barn owls are on average spottier than males in all populations worldwide, directional selection on female spot size (or on genetically correlated traits) may not be restricted to our Swiss population. Thus, why are not all individuals heavily spotted? The observation that mean spot diameter differs substantially among barn owl subspecies (range is 0.45 and $2.65 \mathrm{~mm}$ ) indicates that selection exerted on this trait (or on genetically correlated traits) varies in intensity and is associated with some environmental factors (Roulin et al. 2009). Our results indicate that directional selection on spot diameter was particularly strong during the study period. These arguments suggest that sex ratio bias and sex-specific survival effects may have evolved in other populations. Why did these mechanisms evolve rather than all males being immaculate and all females being marked with large eumelanic spots? Even though spot size is positively selected in females and possibly negatively selected in males, optimal spot size in males may not be zero and a completely black plumage in females may not be optimal. As several genes involved in the production of melanin pigments have pleiotropic effects on a suite of phenotypic traits (Ducrest et al. 2008), suppressing their expression in males may be counter-selected. Thus, displaying spots of different size may advertise sex-specific phenotypic attributes if a polymorphic gene pleiotropically regulates melanogenesis and other phenotypic traits. Identifying these genes and determining their effects is the key next step to understand why selection exerted on spot size or on genetically correlated traits is sexdependent.

\section{ACKNOWLEDGEMENTS}

This work was supported by the Swiss National Science Foundation (to A Roulin and R. Altwegg) and the Norwegian Research Council (to H. Jensen). We thank Pierre Bize, Reto Burri, Philippe Christe, Tim Fawcett, Reinmar Hager, Laurent Keller, Judith Mank, Alexandre 
Reymond, Bernt-Erik Sæther, Valentijn van den Brink, David Westneat and three anonymous referees for comments; Bettina Almasi, Cor Dijkstra, the late Martin Epars and Henri Etter for technical help. The Veterinary service of the 'Canton de Vaud' approved this research.

\section{REFERENCES}

Alonzo, H. \& Sinervo, B. (2007). The effect of sexually antagonistic selection on adaptive sex ratio allocation. Evol. Ecol. Res., 9, $1097-1117$.

Altwegg, R., Roulin, A., Kestenholz, M. \& Jenni, L. (2003). Variation and covariation in survival, dispersal, and population size in barn owls. J. Anim. Ecol., 72, 391-399.

Altwegg, R., Schaub, M. \& Roulin, A. (2007). Age-specific fitness components and their temporal variation in the barn owl. Am. Nat., 169, 47-61.

Blanco, G., Dávila, J.A., López Spetiem, J.A., Rodríguez, R. \& Martínez, F. (2002). Sex-biased initial eggs favours sons in the slightly size-dimorphic scops owls (Otus scops). Biol. J. Linn. Soc., 76, 1-7.

Bonduriansky, R. \& Chenoweth, S.F. (2009). Intralocus sexual conflict. Trends Ecol. Evol., 24, 280-288.

Burnham, K.P. (1993). A theory for combined analysis of ring recovery and recapture data. In: Marked individuals in the study of bird populations (eds Lebreton, J.-D. \& North, P.M.). Birkhäuser Verlag, Basel, pp. 199-213.

Calsbeek, R. \& Sinervo, B. (2002). Uncoupling direct and indirect components of female choice in the wild. Proc. Natl Acad. Sci. USA, 99, 14897-14902.

Calsbeek, R. \& Sinervo, B. (2003). Within-clutch variation in offspring sex determined by differences in sire body size: cryptic mate choice in the wild. J. Evol. Biol., 17, 464-470.

Caswell, H. (2001). Matrix population models. Sinauer, Sunderland, MA.

Charlesworth, B., Coyne, J.A. \& Barton, N.H. (1987). The relative rates of evolution of sex-chromosomes and autosomes. $\mathrm{Am}$. Nat., 130, 113-146.

Chippindale, A.K., Gibson, J.R. \& Rice, W.R. (2001). Negative genetic correlation for adult fitness between the sexes reveals ontogenetic conflict in Drosophila. Proc. Natl Acad. Sci. USA, 98, 1671-1675.

Coulson, T., Kruuk, L.E.B., Tavecchia, G., Pemberton, J.M. \& Clutton-Brock, T.H. (2003). Estimating selection on neonatal traits in red deer using elasticity path analysis. Evolution, 57, 2879-2892.

Day, T. \& Bonduriansky, R. (2004). Intralocus sexual conflict can drive the evolution of genomic imprinting. Genetics, 167, 1537-1546.

Ducrest, A.-L., Keller, L. \& Roulin, A. (2008). Pleiotropy in the melanocortin system, coloration and behavioural syndromes. Trends Ecol. Evol., 23, 502-510.

Ellegren, H. (2007). Molecular evolutionary genomics of birds. Cytogenet. Genome Res., 117, 120-130.

Ellegren, H. \& Parsch, J. (2007). The evolution of sex-biased genes and sex-biased gene expression. Nature Rev. Genet., 8, 689698.
Hadfield, J.D. (2008). Estimating evolutionary parameters when viability selection is operating. Proc. R. Soc. Lond. Ser. B, 275, 723-734.

Hadfield, J.D., Wilson, A.J., Garant, D., Sheldon, B.C. \& Kruuk, L.E.B. (2010). The misuse of BLUP in ecology and evolution. Am. Nat., 175, 116-125.

Jensen, H., Sæther, B.E., Ringsby, T.H., Tufto, J., Griffith, S.C. \& Ellegren, H. (2003). Sexual variation in heritability and genetic correlations of morphological traits in house sparrow (Passer domesticus). J. Evol. Biol., 16, 1296-1307.

Jensen, H., Steinsland, I., Ringsby, T.H. \& Sæther, B.E. (2008). Effects of indirect selection on the evolution of a sexual ornament in the house sparrow (Passer domesticus). Evolution, 62, 1275-1293.

Kruuk, L.E.B. (2004). Estimating genetic parameters in natural populations using the 'animal model'. Philos. Trans. R. Soc. London Ser. B, 359, 873-890.

Lande, R. \& Arnold, S.J. (1983). The measurement of selection on correlated characters. Evolution, 37, 1210-1226.

Lande, R., Engen, S. \& Sæther, B.E. (2003). Stochastic population dynamics in ecology and conservation. Oxford University Press, Oxford.

Lebreton, J.D., Burnham, K.P., Clobert, J. \& Anderson, D.R. (1992). Modeling survival and testing biological hypotheses using marked animals: a unified approach with case studies. Ecol. Monogr., 62, 67-118.

Mank, J.E. (2009). Sex chromosomes and the evolution of sexual dimorphism: lessons from the genome. Am. Nat., 173, 141-150.

Mank, J.E. \& Ellegren, H. (2009). Sex-linkage of sexually antagonistic genes is predicted by female, but not male, effects in birds. Evolution, 63, 1464-1472.

Metz, J.A.J., Nisbet, R.M. \& Geritz, S.A.H. (1992). How should we define 'fitness' for general ecological scenarios? Trends Ecol. Evol., 7, 198-202.

Morris, W.F. \& Doak, D.F. (2002). Quantitative conservation biology. Sinauer Associates Inc., Sunderland, MA.

Mundy, N.I. (2005). A window on the genetics of evolution: MC1R and plumage colouration in birds. Proc. R. Soc. Lond. Ser. B, 272, $1633-1640$.

O’Hara, B., Cano, J.M., Ovaskainen, O., Teplisky, C. \& Alho, J.S. (2008). Bayesian approaches in evolutionary quantitative genetics. J. Evol. Biol., 21, 949-957.

Postma, E. (2006). Implications of the difference between true and predicted breeding values for the study of natural selection and micro-evolution. J. Evol. Biol., 19, 309-320.

R Development Core Team. (2009). R: A language and environment for statistical computing. R Foundation for Statistical Computing.

Rice, W.R. (1984). Sex chromosomes and the evolution of sexual dimorphism. Evolution, 38, 735-742.

Roulin, A. (1999). Nonrandom pairing by male barn owls Tyto alba with respect to a female plumage trait. Behav. Ecol., 10, 688-695.

Roulin, A. (2002). Short- and long-term fitness correlates of rearing conditions in the barn owl. Ardea, 90, 259-267.

Roulin, A. (2004a). Proximate basis of the covariation between a melanin-based female ornament and offspring quality. Oecologia, 140, 668-675.

Roulin, A. (2004b). The evolution, maintenance and adaptive function of genetic colour polymorphism in birds. Biol. Rev., 79, $1-34$. 
Roulin, A. \& Altwegg, R. (2007). Breeding rate is associated with pheomelanism in male and with eumelanism in female barn owls. Behav. Ecol., 18, 563-570.

Roulin, A. \& Dijkstra, C. (2003). Genetic and environmental components of variation in eumelanin and phaeomelanin sextraits in the barn owl. Heredity, 90, 359-364.

Roulin, A., Müller, W., Sasvari, L., Dijkstra, C., Ducrest, A.-L., Riols, C. et al. (2004). Extra-pair paternity, testes size and testosterone level in relation to colour polymorphism in the barn owl Tyto alba. J. Avian Biol., 35, 492-500.

Roulin, A., Wink, M. \& Salamin, N. (2009). Selection on a eumelanic ornament is stronger in the tropics than in temperate zones in the world-wide distributed barn owl. J. Evol. Biol., 22, 345-354.

Sorensen, D.A., Wang, C.S., Jensen, J. \& Gianola, D. (1994). Bayesian inference in threshold models using Gibbs sampling. Genet. Sel. Evol., 26, 333-360.

Spiegelhalter, D.J., Best, N.G., Carlin, B.P. \& van der Linde, A. (2002). Bayesian measures of model complexity and fit. J. $R$. Stat. Soc. B., 64, 583-639.

Steinsland, I. \& Jensen, H. (in press). Utilizing Gaussian Markov Random Field properties of Bayesian animal models. Biometrics (DOI: 10.1111/j.1541-0420.2009.01336.x).

Van Tienderen, H. (2000). Elasticities and the link between demographic and evolutionary dynamics. Ecology, 81, 666-679.

Westneat, D. \& Sih, A. (2009). Sexual conflict as a partitioning of selection. Biol. Lett., 5, 675-677.

White, G.C. \& Burnham, K.P. (1999). Program MARK: survival estimation from populations of marked animals. Bird Study, 46, 120-139.

\section{SUPPORTING INFORMATION}

Additional Supporting Information may be found in the online version of this article:

Table S1 Summary of model selection for barn owl survival analysis.

Table S2 Comparison of Bayesian animal models for estimation of heritability of spot size in barn owls.

Appendix S1 Detailed description of methods.

As a service to our authors and readers, this journal provides supporting information supplied by the authors. Such materials are peer-reviewed and may be re-organized for online delivery, but are not copy-edited or typeset. Technical support issues arising from supporting information (other than missing files) should be addressed to the authors.

Editor, David Westneat

Manuscript received 20 November 2009

First decision made 17 December 2009

Second decision made 16 January 2010

Third decision made 19 January 2010

Manuscript accepted 20 January 2010 